Dalila Bertanha Uhlmann ${ }^{\text {a }}$ (iD) https://orcid.org/0000-0003-4357-6045

Neusa Maria Costa Alexandre (iD) https://orcid.org/0000-0001-5005-3360

Roberta Cunha Matheus Rodrigues (iD) https://orcid.org/0000-0002-6637-7893

Thaís Moreira São-joão

(iD) https://orcid.org/0000-0002-8520-6483

anniversidade Estadual de Campinas (Unicamp), Faculdade de Enfermagem. Campinas, SP, Brasil.

Contato:

Dalila Bertanha UhImann

E-mail:

dalilafisio@yahoo.com.br

Artigo baseado na tese de doutorado de Dalila Bertanha Uhlmann, intitulada Retorno ao trabalho de pacientes com síndrome coronariana aguda (SCA), defendida junto ao Programa de Pós-Graduação em Enfermagem da Faculdade de Enfermagem Universidade Estadual de Campinas em 2017.

As autoras declaram que o estudo recebeu auxílio por meio de bolsa CAPES-DS (Processo no. 01P-377/2017) e que não há conflitos de interesses.

As autoras informam que o trabalho não foi apresentado em reunião científica.

\section{Retorno ao trabalho de pacientes com Síndrome Coronariana Aguda}

\author{
Acute Coronary Syndrome patients' return to work
}

\section{Resumo}

Introdução: as doenças cardiovasculares representam grande impacto de morbimortalidade para a sociedade brasileira. Objetivo: verificar o tempo para retorno ao trabalho de pacientes com Síndrome Coronária Aguda (SCA); identificar e correlacionar variáveis sociodemográficas, clínicas, psicossociais e ocupacionais que podem influenciar nesse período e no desempenho no trabalho. Métodos: estudo transversal correlacional desenvolvido em 20152016 com 65 pacientes com SCA que retornaram ao trabalho. Instrumentos utilizados: Questionário de Caracterização; Questionário de Avaliação do Desempenho no Trabalho; Veterans Specific Activity Questionnaire; MacNew Heart Disease Health-related Quality of Life; Escala Hospitalar de Ansiedade e Depressão; e Impacto da Doença no Cotidiano do Valvopata. Foram aplicados: o teste de Mann-Whitney; pós-teste de Dunn; e coeficiente de correlação de Spearman. Resultados: o tempo médio para retorno ao trabalho foi de 7,3 meses. Os participantes que exerciam trabalho manual apresentaram tempo maior para retorno ao trabalho. O desempenho no trabalho foi fortemente correlacionado com a qualidade de vida e inversamente com ansiedade e depressão. Conclusão: os dados apontam a necessidade de desenvolvimento de estratégias a fim de promover o retorno ao trabalho de pacientes com SCA.

Palavras-chaves: retorno ao trabalho; qualidade de vida; ansiedade; depressão; síndrome coronariana aguda.

\begin{abstract}
Introduction: cardiovascular diseases represent a major morbidity and mortality impact on the Brazilian society. Objective: to verify the Acute Coronary Syndrome (ACS) patients' time to return to work; and to identify and correlate sociodemographic, clinical, psychosocial, and occupational variables that may influence that time and their work performance. Methods: cross-sectional correlational study conducted in 2015-2016 with 65 ACS patients who returned to work. The instruments used were: Sociodemographic Questionnaire; Work Performance Evaluation Questionnaire; Veterans Specific Activity Questionnaire; MacNew Heart Disease Health-related Quality of Life; Hospital Anxiety and Depression Scale; and Impact of Valvular Disease in Everyday Life. Mann-Whitney test, Dunn's posttest, and Spearman's correlation coefficient were applied. Results: participants took 7.3 months on average to return to work; the ones who performed manual labor took longer to do so. Professional performance was found to be strongly correlated with quality of life and, inversely, with anxiety and depression. Conclusion: data indicate the need to develop strategies to promote ACS patients' return to work.
\end{abstract}

Keywords: return to work; quality of life; anxiety, depression, acute coronary syndrome. 


\section{Introdução}

Doenças cardiovasculares (DCVs) representam a principal causa de óbito no mundo e se encontram entre as doenças de maior impacto financeiro ${ }^{1}$. Pessoas com DCVs que residem em países de baixa e média renda per capita têm menos acesso a serviços de saúde eficazes e equitativos ${ }^{1}$, sobretudo os que visam a reabilitação e agilizar o retorno ao trabalho.

A literatura mostra que variáveis sociodemográficas, clínicas e ocupacionais podem influenciar o retorno após eventos cardíacos ${ }^{2-5}$. Entretanto, no Brasil, não foram encontradas pesquisas recentes que analisam essa influência em pacientes com síndrome coronariana aguda (SCA).

A licença médica por doença arterial coronária (DAC) não é a mais frequente, porém, é uma das mais longas ${ }^{5}$. Soma-se a isso o fato de essa afecção ter repercussões negativas nos aspectos físico, emocional, social e econômico, e na qualidade de vida relacionada à saúde (QVRS) ${ }^{4,5}$, que pode afetar o cotidiano laboral dos pacientes. Sendo assim, a assistência a esses pacientes deve incluir cuidados relacionados às variáveis psicossociais ${ }^{6}$.

A necessidade de tratamentos clínico e cirúrgico pode interferir no retorno do indivíduo a suas atividades de rotina. Além disso, a capacidade para o trabalho - um construto que indica, como qualificador, o nível máximo possível de funcionalidade que uma pessoa consegue atingir ${ }^{7-9}$ - pode estar alterada no retorno, devendo, por esse motivo, ser avaliada ${ }^{9}$.

A fim de conhecer e propor intervenções e sugerir pesquisas que visem diminuir o tempo de retorno ao trabalho e reduzir os custos relacionados, para o indivíduo e para a sociedade, os objetivos deste estudo foram: verificar o tempo para retorno ao trabalho de pacientes com SCA; identificar e correlacionar variáveis sociodemográficas, clínicas, psicossociais e ocupacionais que podem influenciar no tempo para retorno e no desempenho no trabalho.

\section{Métodos}

Estudo transversal exploratório correlacional realizado em Ambulatório Especializado de Cardiologia de um hospital público universitário no município de Campinas, São Paulo, que atende a usuários do sistema de saúde pública e que não dispõe de serviço de reabilitação cardíaca. O estudo foi aprovado pelo Comitê local de Ética em Pesquisa (Parecer $\left.\mathrm{n}^{\mathrm{o}} 611.105 / 2014\right)$.

Tendo em vista que os pacientes isquêmicos são seguidos em serviços especializados de nível secundário, o que limita o acesso a essa população, e considerando o número restrito dos que se encontram em seguimento clínico que retornam ao trabalho, a amostragem se deu por conveniência, constituída pela inclusão sequencial dos pacientes, entre novembro de 2015 a abril de 2016. Os pacientes com SCA foram identificados por meio do prontuário e convidados a participar da pesquisa, no dia da consulta médica agendada no ambulatório de cardiologia. Assim, foram incluídos 65 indivíduos com diagnóstico de SCA que relataram estar trabalhando. Foram excluídos os pacientes que, após serem avaliados quanto ao estado cognitivo por meio de questionário contendo sete perguntas pré-estabelecidas e adaptadas ${ }^{10}$, não responderam corretamente a pelo menos cinco questões.

A entrevista foi feita individualmente e realizada pela pesquisadora principal, em local privativo, com os questionários descritos a seguir.

\section{Instrumento de caracterização sociodemográfica, clínica e ocupacional}

Foi utilizado um instrumento de caracterização sociodemográfica, clínica e ocupacional, construído e validado em estudo pregresso ${ }^{11,12}$, com algumas adaptações. Optou-se também usar o questionário para obter informações sobre o impacto de determinadas variáveis clínicas tais como diagnóstico, sintomas e comorbidades no retorno ao trabalho. O tipo de ocupação foi classificado conforme a proposta de Hébert ${ }^{13}$ em: "trabalho manual" - atividade que envolve manipulação de cargas pesadas e médias por um período de tempo contínuo, e/ou cargas mais leves, mas com uso de postura estática contínua e/ou trabalho repetitivo contínuo; "trabalho misto" - envolve a manipulação de cargas leves e postura estática descontínua ou ocasionalmente carga pesada ou média (também são inclusos nesta categoria aqueles que exigem muitos movimentos e manipulações com carga leve e realizam movimentos repetitivos); e "trabalho não manual” - aquele no qual o esforço físico é mínimo.

Foram considerados ativos os pacientes que relataram estar trabalhando após o evento isquêmico e os aposentados e/ou afastados, mas que desenvolviam alguma atividade de trabalho. Foram considerados inativos os pacientes aposentados compulsoriamente e/ou por invalidez e os afastados por auxílio-doença.

Foi definido como tempo para retorno ao trabalho o período transcorrido entre a data do evento isquêmico agudo e a data em que o indivíduo retornou ao trabalho. 


\section{Questionário de Avaliação do Desempenho no Trabalho (WRFQ-Br)}

O Work Role Functioning Questionnaire (WRFQ) foi traduzido e validado para a língua portuguesa do Brasil ${ }^{9}$. O objetivo deste instrumento é avaliar a capacidade funcional do trabalhador em relação ao impacto e limitações decorrentes de afecções crônicas nas atividades de trabalho diárias. É composto por cinco dimensões de demandas: de plano de trabalho, físicas, mentais, sociais e de produção. O cálculo do escore total gera um índice que varia entre $0 \%$ e $100 \%$, que reflete a capacidade para o trabalho do indivíduo de acordo com as funções. Quanto maior o índice, melhor a capacidade para o trabalho ${ }^{9}$.

\section{Versão brasileira do Veterans Specific Activity Questionnaire (VSAQ)}

O VSAQ é um questionário breve, elaborado para determinar o nível máximo de atividade física diária que uma pessoa pode fazer de acordo com a ocorrência de sintomas cardiovasculares. Este instrumento foi adaptado para a cultura brasileira e validado para $\mathrm{DCV}^{14}$, tendo apresentado propriedades de medida satisfatórias. Consiste em uma lista de atividades elencadas em ordem progressiva, conforme o Equivalente Metabólico de Tarefa (MET). No estudo de validação do $\mathrm{VSAQ}^{14}$, foi constatado que a inclusão da idade do paciente no escore melhora a capacidade de prever a tolerância ao exercício. Dessa forma, foi proposto um nomograma a ser aplicado de acordo com a pontuação obtida pelo VSAQ, expresso pela equação: $\mathrm{METs}=4,7+0,97$ (VSAQ) - 0,06 (id ade). O VSAQ tem sido considerado adequado no acompanhamento regular de pacientes com DCV para avaliar o impacto da doença na vida diária e o nível de tolerância do paciente ao exercício ${ }^{14}$.

\section{Versão brasileira do MacNew Heart Disease Health- related Quality of Life (MacNew)}

Consiste na versão modificada do instrumento original Quality of Life after Myocardial Infarction (QLMI), originalmente desenvolvido para avaliar a QVRS de pacientes com infarto do miocárdio ${ }^{15}$. A versão brasileira do MacNew é composta por 27 itens distribuídos em três domínios: função física, função emocional e função social. O escore máximo possível em qualquer domínio é 7 (melhor QVRS) e o mínimo é 1 (pior QVRS). O escore de QVRS global pode ser calculado por meio da soma da média de todos os itens ${ }^{16}$.

\section{Versão brasileira da Escala Hospitalar de Ansiedade e Depressão (Hads)}

A Hads foi validada para a língua portuguesa do Brasil $^{17}$. Embora tenha sido inicialmente proposta para pacientes ambulatoriais na detecção de estados depressivos e de ansiedade, pode ser aplicada em diversos contextos ${ }^{18}$. A escala contém 14 questões de múltipla escolha e é composta por duas subescalas: ansiedade (Hads-A) e depressão (Hads-D), com sete itens em cada domínio ${ }^{17,19}$. A pontuação global em cada subescala varia de 0 a 21 . Para a interpretação dos valores das duas subescalas, considera-se que quanto maior a pontuação, maior a chance de desenvolver um transtorno de ansiedade e/ou de depressão ${ }^{19}$. Outra forma de análise é considerar como critério um escore 8 como ponto de corte ${ }^{19}$.

\section{Impacto da Doença no Cotidiano do Valvopata (IDCV)}

O instrumento IDCV foi desenvolvido originalmente para avaliação de crenças de pacientes valvopatas $^{20}$, mas constatou-se, em estudo posterior ${ }^{21}$, que o conjunto de afirmativas que o compõe é capaz de avaliar o impacto da cronicidade imposta pela cardiopatia. É constituído por duas partes: Parte A - composta por 14 itens que medem as percepções relativas ao impacto da doença; Parte B - com 14 itens que medem a avaliação que pessoa faz sobre cada consequência mencionada na primeira escala, quer ela ocorra ou não em sua vida. Os itens estão agrupados em quatro domínios: impacto físico da doença - sintomas; impacto da doença nas atividades cotidianas; impacto social e emocional da doença; e adaptação à doença. Para determinar o escore final do IDCV é necessário transformar todos os itens na mesma direção. O escore total é calculado pela soma de todos os produtos obtidos, sendo possível uma variação entre 14 a 350. Quanto maior o escore, maior o impacto negativo percebido pelo indivíduo ${ }^{20}$.

\section{Análise estatística}

Os dados foram inseridos em uma planilha eletrônica no software Statistical Package for Social Sciences (SPSS), versão 22.0 para Windows; e transferidos para o programa Statistical Analysis System for Windows, versão 9.4 (Statistical Analysis System - SAS Institute Inc., Cary, NC, USA, 2008), para realização das seguintes análises:

- Descritiva: com confecção de tabelas de frequência, medidas de posição (média, mediana, mínima e máxima) e dispersão (desvio padrão) para dados de caracterização sociodemográfica, clínica e ocupacional e para domínios e escore total dos instrumentos de avaliação do desempenho no trabalho, aptidão cardiorrespiratória, QVRS, ansiedade, depressão e impacto da doença.

- Comparação: foi empregado o teste não paramétrico de Mann-Whitney ${ }^{22}$, para comparação 
entre o tempo para retorno do trabalho e as variáveis clínicas (sintomas, condições clínicas associadas e hábitos de vida) e variável ocupacional (tipo de trabalho). O Pós-teste de Dunn ${ }^{22}$ foi utilizado para comparar o tempo de retorno do trabalho com o tipo de atividade exercida.

- Correlação: foi utilizado o coeficiente de correlação de Spearman ${ }^{22}$ para verificar a existência de correlação entre o tempo para retorno ao trabalho e a escolaridade, a renda familiar, o tempo no emprego atual, a carga horária, a avaliação do desempenho no trabalho, a aptidão cardiorrespiratória, a QVRS, a ansiedade, a depressão e o impacto da doença. Também foi utilizado o coeficiente de correlação de Spearman para correlacionar o desempenho no trabalho com a aptidão cardiorrespiratória (VSAQ), a QVRS na coronariopatia (MacNew), a ansiedade e a depressão (HADS) e o impacto da doença (IDCV).

Optou-se pela seguinte classificação para avaliar a magnitude das correlações: 0,1 a 0,29 (fraca), 0,30 a 0,49 (moderada) e maior ou igual a 0,50 (forte) ${ }^{23}$. Foi adotado nível de significância menor que 5\%.

\section{Resultados}

A análise descritiva da amostra evidenciou maioria de homens $(90,7 \%)$, casados $(70,7 \%)$, com média de idade de 56 anos, escolaridade média de 7,3 anos, renda familiar média de 3,2 salários mínimos. Em relação às variáveis clínicas, a maioria apresentou diagnóstico de IAM $(93,8 \%)$, relatou precordialgia (38,4\%), palpitação $(41,5 \%)$ e dispneia $(43,8 \%)$ no mês que antecedeu a entrevista. A condição clínica associada mais frequente foi a hipertensão $(81,5 \%)$, seguida pela dislipidemia $(78,4 \%)$. Em relação aos hábitos de vida, o tabagismo $(69,2 \%)$ foi o mais frequente (Tabela 1).

A caracterização ocupacional e análise descritiva do desempenho no trabalho de pacientes com SCA estão apresentados na Tabela 2. Quanto às variáveis ocupacionais, o tempo médio de trabalho após a data do evento isquêmico agudo foi de 2,3 anos; a carga horária média foi de 42,7 horas e o tipo de trabalho foi predominantemente misto $(47,7 \%)$. O tempo para retorno ao trabalho foi, em média, 7,3 meses após a SCA.

Os escores elevados observados em todos os domínios indicam bom índice de desempenho no trabalho.

Os achados da análise descritiva da aptidão cardiorrespiratória, QVRS, ansiedade e depressão e impacto da doença são apresentados na Tabela 3. A aptidão cardiorrespiratória, avaliada pelo VSAQ, apresentou valor médio de 7,9 equivalentes metabólicos de tarefa (MET), o que indica uma moderada tolerância ao exercício. Ao avaliar a QVRS, notaram-se valores similares entre as funções física $(5,7)$, emocional $(5,7)$ e social $(5,8)$, mostrando elevada QVRS, considerando-se que o escore máximo possível é 7,0. Quanto à ansiedade e depressão, cuja variação possível para cada escala é de 0 a 21 e que quanto maior o valor, maior a chance de o indivíduo desenvolver um transtorno de ansiedade e/ou depressão, observou-se que os escores variaram entre $3 \mathrm{e}$ 16 e entre 0 e 15, respectivamente. Verificou-se também maior pontuação na escala de ansiedade $(5,6)$ em relação à depressão $(3,5)$. Ao utilizar o critério sugerido pelos autores da escala ${ }^{19}$, que determina o escore 8 como ponto de corte para a Hads-Ansiedade e a Hads-Depressão, foram constatados valores maiores para Hads-A em $24,6 \%$ dos pacientes e para Hads-D em 21,5\% dos pacientes com SCA. Quanto ao impacto da doença no cotidiano, foi observado um escore total médio de 140,1. Considerando-se que o escore total do IDCV pode variar entre $14 \mathrm{e}$ 350 , e que o escore 175 divide os resultados em duas categorias, baixo impacto (escore total $<175$ ) e alto impacto (escore total $>175$ ), foi constatado que os pacientes se encontravam em uma situação de baixo impacto (Tabela 3).

A comparação entre o tipo de atividade e o tempo para retorno ao trabalho foi avaliada por meio do teste de Mann-Whitney (Tabela 4). O tipo de trabalho foi a única variável que apresentou relação significante com o tempo para retorno ao trabalho, sendo que aqueles que desenvolviam atividade manual relataram tempo para retorno mais elevado do que aqueles cujo trabalho era o não manual.

As análises de correlação foram testadas por meio do coeficiente de correlação de Spearman e são apresentadas na Tabela 5. Não foram constatadas correlações entre o tempo para retorno ao trabalho e as variáveis sociodemográficas (escolaridade, renda familiar, idade), ocupacionais (tempo no emprego e carga horária, WRFQ), clínicas (obesidade - IMC > 30Kg/m2 e aptidão cardiorrespiratória VSAQ) e psicossociais - QVRS (MacNew), ansiedade e depressão (Hads) e impacto da doença no cotidiano do paciente com coronariopatia (IDCV).

Não houve diferença entre o tempo para retorno ao trabalho e os sintomas (precordialgia, palpitação, dispneia), comorbidades (HAS, diabetes, dislipidemia) e os hábitos de vida (tabagismo). Não foi possível comparar as variáveis (sexo, AVE, terapia de reposição hormonal e etilismo) com o tempo para retorno ao trabalho devido ao número reduzido de indivíduos que apresentavam essas variáveis. 
Tabela 1 Caracterização sociodemográfica e clínica de pacientes com síndrome coronariana aguda (N=65) atendidos em serviço ambulatorial de hospital público universitário no período de novembro de 2015 a abril de 2016. Campinas, SP, 2016

\begin{tabular}{|c|c|c|c|c|c|}
\hline Variável & $N$ & $\%$ & Média (DP) & Mediana $(I Q R)$ & Variação \\
\hline \multicolumn{6}{|c|}{ Variáveis sociodemográficas } \\
\hline Idade (em anos) & 65 & 100,0 & $56(8,5)$ & $55(10,0)$ & $29-74$ \\
\hline \multicolumn{6}{|l|}{ Sexo } \\
\hline Masculino & 59 & 90,7 & & & \\
\hline Feminino & 6 & 9,2 & & & \\
\hline Escolaridade (em anos) & & & $7,3(3,7)$ & $08(7,0)$ & $0-15$ \\
\hline Renda familiar (em salários mínimos) & & & $3,2(1,9)$ & $2,5(2,8)$ & $0,9-11,3$ \\
\hline \multicolumn{6}{|l|}{ Estado civil } \\
\hline Casado & 46 & 70,7 & & & \\
\hline Desquitado/divorciado & 8 & 12,3 & & & \\
\hline Solteiro & 5 & 7,6 & & & \\
\hline União consensual & 4 & 6,1 & & & \\
\hline Viúvo & 2 & 3,0 & & & \\
\hline \multicolumn{6}{|l|}{ Raça } \\
\hline Branca & 47 & 72,0 & & & \\
\hline Outras & 18 & 28,0 & & & \\
\hline \multicolumn{6}{|c|}{ Variáveis clínicas } \\
\hline \multicolumn{6}{|l|}{ Diagnóstico principal } \\
\hline Infarto agudo do miocárdio & 61 & 93,8 & & & \\
\hline Angina instável & 4 & 6,1 & & & \\
\hline \multicolumn{6}{|l|}{ Sintomas (no último mês) } \\
\hline Precordialgia & 25 & 38,4 & & & \\
\hline Palpitação & 27 & 41,5 & & & \\
\hline Dispneia & 28 & 43,0 & & & \\
\hline \multicolumn{6}{|l|}{ Comorbidades } \\
\hline Obesidade (IMC > 30 kg/m²) & 16 & 24,6 & & & \\
\hline Hipertensão arterial sistêmica & 53 & 81,5 & & & \\
\hline Diabetes & 20 & 30,7 & & & \\
\hline Dislipidemia & 51 & 78,4 & & & \\
\hline Acidente vascular encefálico (AVE) & 2 & 3,0 & & & \\
\hline Outras & 19 & 29,2 & & & \\
\hline \multicolumn{6}{|l|}{ Hábitos de vida } \\
\hline Tabagismo (pregresso e atual) & 45 & 69,2 & & & \\
\hline Etilismo (pregresso) & 30 & 20,7 & & & \\
\hline Faz acompanhamento médico para outras doenças & 15 & 23,0 & & & \\
\hline
\end{tabular}

DP: desvio padrão.

IQR: Interquartile range (Intervalo interquartil-Q3-Q1). 
Tabela 2 Caracterização ocupacional e análise do desempenho no trabalho de pacientes com síndrome coronariana aguda $(\mathrm{N}=65)$ atendidos em serviço ambulatorial de hospital público universitário, no período de novembro de 2015 a abril de 2016. Campinas, SP, 2016

\begin{tabular}{|c|c|c|c|c|c|}
\hline Variável ocupacional & $N$ & $\%$ & Média (DP) & Mediana $(I Q R)$ & Variação \\
\hline Tempo que trabalha no emprego atual (em anos) & & & $12,1(14,1)$ & $6(18,4)$ & $0,08-54$ \\
\hline Carga horária (horas) & & & $42,7(14,3)$ & $40(12,0)$ & $16-98$ \\
\hline Tempo de retorno ao trabalho após SCA (em meses) & & & $7,3(12)$ & $3(5,0)$ & $0,1-65$ \\
\hline Tempo de trabalho após a SCA (em anos) & & & $2,3(2,9)$ & $1,08(2,4)$ & $0,08-12,4$ \\
\hline \multicolumn{6}{|l|}{ Tipo de trabalho } \\
\hline Manual & 19 & 29,2 & & & \\
\hline Não manual & 15 & 23,0 & & & \\
\hline Misto & 31 & 47,6 & & & \\
\hline \multicolumn{6}{|l|}{ Esteve afastado nos últimos 12 meses? } \\
\hline Não & 43 & 66,1 & & & \\
\hline Sim & 22 & 33,8 & & & \\
\hline \multicolumn{6}{|l|}{ WRFQ - Domínios } \\
\hline Plano trabalho & & & $87,9(18,9)$ & $85,0(15,0)$ & $5-100$ \\
\hline Demanda física & & & $87,3(16,7)$ & $87,5(8,3)$ & $29,1-100$ \\
\hline Demanda mental & & & $91,5(13,1)$ & $91,6(8,3)$ & $41,6-100$ \\
\hline Demanda social & & & $92,9(9,1)$ & $91,6(8,3)$ & $66,6-100$ \\
\hline Demanda de produção & & & $86,9(15,2)$ & $82,1(14,2)$ & $29,1-100$ \\
\hline Escore total & & & $88,7(13,1)$ & $87,5(10,3)$ & $88,7-100$ \\
\hline
\end{tabular}

DP: desvio padrão.

IQR: Interquartile range (Intervalo interquartil - Q3-Q1).

WRFQ: Questionário de Avaliação do Desempenho no Trabalho.

Tabela 3 Análise da aptidão cardiorrespiratória, qualidade de vida relacionada à saúde, ansiedade, depressão e impacto da doença no cotidiano dos pacientes com síndrome coronariana aguda, $(\mathrm{N}=65)$ atendidos em serviço de hospital público universitário, no período de novembro de 2015 a abril de 2016. Campinas, SP, 2016

\begin{tabular}{|c|c|c|c|}
\hline Variável & Média (DP) & Mediana $\left(I Q R^{*}\right)$ & Variação observada \\
\hline VSAQ (Mets) & $7,9(2,3)$ & $6,2(3,1)$ & $3,3-14,2$ \\
\hline \multicolumn{4}{|l|}{ MacNew - Domínios } \\
\hline Função física & $5,7(0,9)$ & $5,2(1,1)$ & $3-7$ \\
\hline Função emocional & $5,7(0,9)$ & $5,2(1,2)$ & $2,7-7$ \\
\hline Função social & $5,8(0,9)$ & $5,4(0,9)$ & $2,3-7$ \\
\hline Escore global & $5,6(0,8)$ & $5,2(1,0)$ & $3-6,8$ \\
\hline \multicolumn{4}{|l|}{ Hads - Escalas } \\
\hline Hads-Ansiedade & $5,6(4,2)$ & $3,0(4,0)$ & $3-16$ \\
\hline Hads-Depressão & $3,5(4,2)$ & $0,0(6,0)$ & $0-15$ \\
\hline \multicolumn{4}{|l|}{ IDCV - Domínios } \\
\hline Impacto físico da doença - Sintomas & $30,4(21,3)$ & $25,0(39,0)$ & $6-75$ \\
\hline Impacto da doença nas atividades cotidianas & $55,4(29,4)$ & $55,0(48,0)$ & $8-120$ \\
\hline Impacto social e emocional da doença & $39,6(22,8)$ & $38,0(38,0)$ & $6-100$ \\
\hline Adaptação à doença & $14,6(8,3)$ & $14,0(15,0)$ & $3-30$ \\
\hline Escore total & $140,1(66,3)$ & $125,0(103,0)$ & $35-320$ \\
\hline
\end{tabular}

*Interquartile range (Intervalo interquartil - Q3-Q1).

VSAQ (Mets): Veterans Specific Activity Questionnaire.

MacNew: MacNew Heart Disease Health-related Quality of Life instrument.

Hads: Escala Hospitalar de Ansiedade e Depressão.

IDCV: Questionário de Impacto da Doença no Cotidiano do Valvopata. 
Tabela 4 Comparação entre o tipo de trabalho e tempo de retorno ao trabalho de pacientes com síndrome coronariana aguda $(\mathrm{N}=65)$ atendidos em serviço de hospital público universitário, no período de novembro de 2015 a abril de 2016. Campinas, SP, 2016

\begin{tabular}{ccccccc}
\hline Variável & Tipo de trabalho & $N$ & Média (DP) & Mediana (IQR) & Variação $^{\text {p-valor }}{ }^{*}$ \\
\hline Tempo de retorno & Manual & 19 & $9,9(12,6)$ & $3,0(9,9)$ & $0,50-48,0$ & 0,0348 \\
& Não manual & 15 & $5,0(12,0)$ & $1,0(2,5)$ & $0,10-48,0$ \\
& Misto & 31 & $6,8(11,8)$ & $4,0(3,9)$ & $0,50-65,0$ & \\
\hline
\end{tabular}

"p-valor <0,05; 'Teste de Mann-Whitney.

Os resultados das análises de correlação entre a medida de desempenho no trabalho e as variáveis clínicas (aptidão cardiorrespiratória) e variáveis psicossociais (QVRS, ansiedade, depressão impacto da doença no cotidiano) estão apresentadas na Tabela 5. Os resultados mostram que a maioria dos domínios do WRFQ-Br, que avalia o desempenho no trabalho, apresentou correlações significantes positivas de forte magnitude com os domínios do MacNew, exceto pelo domínio
Demanda social do WRFQ. O desempenho no trabalho também apresentou correlações negativas significantes de moderada a forte magnitude com as medidas de ansiedade e depressão; mostrando que quanto melhor o desempenho, menor o traço de ansiedade e depressão. Por outro lado, o desempenho foi fracamente correlacionado com a aptidão cardiorrespiratória e não apresentou correlação com o escore total do impacto da doença no cotidiano (IDCV).

Tabela 5 Correlação de Spearman ( $\rho$ ) entre as medidas de desempenho no trabalho e aptidão cardiorrespiratória, QVRS, ansiedade, depressão e impacto da doença no cotidiano de pacientes com síndrome coronariana aguda $(\mathrm{N}=65)$ atendidos em serviço de hospital público universitário, no período de novembro de 2015 a abril de 2016. Campinas, SP, 2016

\begin{tabular}{|c|c|c|c|c|c|c|}
\hline \multirow[b]{2}{*}{ Variável } & \multicolumn{6}{|c|}{ WRFQ-Br-Domínios } \\
\hline & $\begin{array}{c}\text { Plano } \\
\text { trabalho }\end{array}$ & $\begin{array}{l}\text { Demanda } \\
\text { física }\end{array}$ & $\begin{array}{c}\text { Demanda } \\
\text { mental }\end{array}$ & $\begin{array}{c}\text { Demanda } \\
\text { social }\end{array}$ & $\begin{array}{c}\text { Demanda } \\
\text { de produção }\end{array}$ & $\begin{array}{c}\text { Escore } \\
\text { total }\end{array}$ \\
\hline VSAQ (Mets) & 0,19 & $0,25^{*}$ & $0,26^{*}$ & 0,13 & 0,19 & $0,27^{*}$ \\
\hline \multicolumn{7}{|l|}{ MacNew-Domínios } \\
\hline Função física & $0,75^{\ddagger}$ & $0,67^{\sharp}$ & 0,64 & $0,39^{\dagger}$ & $0,75^{\ddagger}$ & $0,83^{\ddagger}$ \\
\hline Função emocional & 0,74 & $0,65^{\ddagger}$ & $0,61^{\ddagger}$ & $0,41^{\dagger}$ & $0,67^{*}$ & $0,78^{\ddagger}$ \\
\hline Função social & $0,60^{\ddagger}$ & $0,56^{*}$ & 0,59 & $0,41^{\dagger}$ & $0,70^{\ddagger}$ & $0,73^{\ddagger}$ \\
\hline Escore total & $0,77^{\ddagger}$ & $0,68^{*}$ & $0,64 \stackrel{\ddagger}{\ddagger}$ & $0,41^{\dagger}$ & 0,74 & $0,83^{\ddagger}$ \\
\hline \multicolumn{7}{|l|}{ Hads- Escalas } \\
\hline Hads - Ansiedade & $-0,66^{\ddagger}$ & $-0,46^{\ddagger}$ & $-0,57 \ddagger$ & $-0,38^{\dagger}$ & $-0,62$ & $-0,68^{\ddagger}$ \\
\hline Hads - Depressão & $-0,69$ & $-0,63^{\sharp}$ & $-0,58^{\ddagger}$ & $-0,51^{*}$ & $-0,64$ & $-0,75^{\ddagger}$ \\
\hline \multicolumn{7}{|l|}{ IDCV- domínios } \\
\hline Impacto físico & 0,02 & $-0,04$ & 0,04 & $-0,02$ & 0,02 & 0 \\
\hline $\begin{array}{l}\text { Impacto da doença no } \\
\text { cotidiano }\end{array}$ & 0,16 & 0,12 & 0,03 & 0,04 & 0,12 & 0,1 \\
\hline Impacto social e emocional & 0,18 & 0,13 & 0,09 & 0,14 & 0,17 & 0,13 \\
\hline Adaptação à doença & $0,37^{\dagger}$ & $0,31^{\dagger}$ & $0,31^{*}$ & 0,26 & 0,28 & $0,39^{\dagger}$ \\
\hline Escore Total & 0,15 & 0,11 & 0,07 & 0,08 & 0,11 & 0,1 \\
\hline
\end{tabular}

$*<0,05,{ }^{\dagger}<0,01, \stackrel{\star}{*}<0,001$;

WRFQ -Br: Questionário de Avaliação do Desempenho No Trabalho.

VSAQ (METS): Veterans Specific Activity Questionnaire.

MacNew: MacNew Heart Disease Health-related Quality of Life instrument.

Hads: Escala Hospitalar de Ansiedade e Depressão.

IDCV: Questionário de Impacto da Doença no Cotidiano do Valvopata. 


\section{Discussão}

Este estudo procurou conhecer determinadas interfaces do retorno ao trabalho em pacientes com SCA. Em relação às características sociodemográficas, participaram pacientes com média de idade de 56 anos. Pesquisas sugerem que quanto menor a idade maior a chance de retorno ao trabalho em pacientes com infarto agudo do miocárdio (IAM) ${ }^{3}$.

O nível médio de escolaridade foi de 7,3 anos, sendo maior do que aqueles encontrados em outros estudos $^{6,24}$. A renda familiar média foi de 3,2 salários mínimos, superior à encontrada em estudos prévios $^{6,25}$ conduzidos na mesma instituição, o que pode ser explicado porqueenvolveram indivíduos com cardiopatia ativos e inativos. Indivíduos mais jovens e com maior escolaridade que relatam boas condições de saúde e alta QVRS também retornam a suas atividades profissionais com maior frequência ${ }^{5}$.

O tempo médio de retorno ao trabalho foi de 7,3 meses, tempo superior aos encontrados em outros estudos, que identificaram que a maioria dos pacientes com SCA retorna em até seis meses ${ }^{2,4}$. $\mathrm{O}$ retorno ao trabalho tem sido um grande desafio para a saúde do trabalhador. Fatores como dor, questões psicossociais, demanda psicológica do trabalho, apoio organizacional, programas de retorno ao trabalho, modificações nos postos de trabalho e políticas de apoio mostraram-se na literatura como facilitadores e/ou barreiras de retorno ao trabalho e precisam ser observados neste processo ${ }^{2-5,26}$. Algumas discussões precisam ser aprofundadas, principalmente no que se refere às diferenças de retorno ao trabalho entre pessoas com diferentes atividades ${ }^{26}$.

No Brasil, poucos são os estudos relacionados à temática "retorno ao trabalho" e não se encontrou pesquisa sobre esse tema para o paciente com SCA. Considerado um país em desenvolvimento, em que as DCV representam grande porcentagem de despesas na economia ${ }^{1}$ e que a licença médica por DCV é uma das mais longas ${ }^{5}$, é preocupante o fato de que poucas estratégias estejam sendo adotadas para lidar com essa situação, a fim de diminuir o tempo para retorno ao trabalho.

Em relação às variáveis ocupacionais, os tipos de trabalho mais frequentes foram o misto e o manual, e a carga horária média semanal foi de 42,7 horas. $\mathrm{Na}$ avaliação do WRFQ-Br, a demanda de produção, que está relacionada à produtividade e à qualidade de serviço, apresentou menor valor. Os indivíduos profissionalmente ativos que tiveram IAM têm um grau variável de readaptação ao trabalho ${ }^{5}$.
O VSAQ foi utilizado para estimar a aptidão cardiorrespiratória, baseado em sintomas percebidos durante as atividades diárias. Destaca-se estudo com 95 pacientes cardiopatas estáveis, assintomáticos, sem anormalidades eletrocardiográficas, em que foi constatado 4,9 Mets como aptidão cardiorrespiratória média ${ }^{27}$, valor inferior ao encontrado no presente estudo. Este fato pode ser explicado pela inclusão de indivíduos ativos e não ativos. Neste estudo, também se observou moderado escore na avaliação da VSAQ considerando a idade, indicando moderada tolerância ao exercício.

Em relação à QVRS, observou-se escore total médio de 5,6, mostrando uma elevada QVRS. Os valores médios dos domínios e do escore total da QVRS foram maiores do que em outros estudos com pacientes com coronariopatia ativos e inativos ${ }^{28-30}$. Isto pode ser causado em razão de os indivíduos que estavam trabalhando apresentarem classificação menos grave da doença.

Quanto à ansiedade e depressão, observa-se que os valores médios foram menores em relação a estudo ${ }^{29}$ realizado com 112 pacientes com IAM; outra pesquisa ${ }^{3}$, com 48 pacientes com IAM que haviam retornado ao trabalho, observou valores médios para depressão de 3,6 na escala Hads, similares aos aqui encontrados. O mesmo estudo ${ }^{3}$ não encontrou diferenças quanto à ansiedade e depressão em indivíduos que haviam retornado a suas ocupações em relação aos que não estavam trabalhando. Ao considerar o ponto de corte sugerido pelo autor da escala ${ }^{19}$, observa-se que houve uma ocorrência elevada de trabalhadores com escores superiores a 8 na escala Hads, no presente estudo, em ambas as subescalas de ansiedade e depressão, indicando a necessidade de ações curativas e preventivas.

Ao analisar o impacto da doença cardíaca no cotidiano, observou-se, pelo valor médio, que os pacientes se encontravam em situação de baixo impacto. Estudo realizado com 135 pacientes com insuficiência cardíaca evidenciou que a média do escore total do instrumento IDCV foi de $173,1^{31}$, valor superior ao encontrado no presente estudo $(140,1)$. A inclusão de pacientes ativos e não ativos pode ter relação com o achado da pesquisa anterior. Seria relevante o desenho de novos estudos que comparassem os indivíduos com cardiopatia, em diferentes situações de trabalho, a fim de verificar o impacto da doença cardíaca nestas distintas situações.

Os dados do nosso estudo sugerem que os indivíduos que exerciam trabalho manual apresentaram tempo para retorno ao trabalho maior. Este resultado era esperado, considerando que os sintomas 
relacionados à DAC estão fortemente relacionados aos esforços físicos. As demais variáveis sociodemográficas e clínicas não influenciaram o tempo para retorno ao trabalho Outros estudos também mostram que variáveis clínicas parecem ter pouca relevância para o retorno ao trabalho ${ }^{2}$.

Esta pesquisa, ao correlacionar o desempenho no trabalho com a aptidão cardiorrespiratória, QVRS, ansiedade, depressão e o impacto da doença cardiovascular, procurou fornecer subsídios para o planejamento de intervenções que visam o retorno ao trabalho das pessoas acometidas com SCA.

Ao considerar a aptidão cardiorrespiratória do paciente com SCA, verificou-se que foi fracamente correlacionada com o desempenho no trabalho. Estudo sobre retorno ao trabalho encontrou que desempenhar pouca atividade física após o IAM pode influenciar negativamente esse retorno ${ }^{3}$. Os programas de reabilitação cardíaca devem incluir avaliação ocupacional envolvendo as várias facetas que compõem o desempenho no trabalho.

Em relação à QVRS, sabe-se que, além de perda de dias trabalhados e prejuízos para o setor produtivo, a DAC pode influenciar a QVRS dos pacientes $^{3}$. O IAM pode levar ao comprometimento de aspectos físicos, psicológicos e sociais, causando grande impacto na QVRS $^{5}$. Verificou-se que indívíduos que sofreram IAM e retornaram ao trabalho apresentaram não apenas a melhora da QVRS, mas também benefícios econômicos, individuais e para a sociedade ${ }^{3}$. O presente estudo mostrou que o desempenho no trabalho está fortemente correlacionado com a QVRS.

Esta pesquisa mostrou, ainda, que pacientes com melhor desempenho no trabalho apresentam menores traços de ansiedade e depressão. A depressão é comum em pacientes com IAM e contribui para dificuldades com a retomada ao trabalho ${ }^{32}$. Assim, intervenções que envolvem não somente o retorno trabalho, mas também programas direcionados à saúde do trabalhador, particularmente em relação aos distúrbios de ansiedade e depressão de indivíduos com SCA, podem facilitar o retorno ao trabalho.

Não foi estabelecida relação entre o desempenho no trabalho e a avaliação do impacto da doença. Este fato pode sugerir que indivíduos com SCA que retornaram a suas atividades ocupacionais não apresentam percepção de consequências da doença no cotidiano.

Quanto às limitações deste estudo, pode-se citar problemas em realizar o acompanhamento dos pacientes após evento cardíaco por causa das dificuldades de retorno ao serviço de saúde, relacionadas às questões socioeconômicas, de transporte e ligadas à própria organização do sistema de saúde. Destacam-se também os vieses das medidas autorrelatadas, como a desejabilidade social e o viés de memória. O paciente com SCA foi caracterizado segundo o diagnóstico, sintomas, comorbidades, idade, sexo e aptidão cardiorrespiratória, entretanto, seria interessante a realização de estudos futuros com desenho longitudinal para verificar na população brasileira o impacto do tratamento da SCA no retorno ao trabalho. Recomenda-se a condução de estudos futuros com o emprego da análise de sobrevivência, com vistas a avaliar o desfecho da doença em variáveis psicossociais, como o retorno ao trabalho.

\section{Conclusão}

Os achados deste estudo sugerem que os indivíduos com SCA que exercem trabalho manual têm tempo prolongado de retorno ao trabalho. Constatou-se que o desempenho no trabalho foi fortemente correlacionado com a QVRS e inversamente correlacionado com a depressão e ansiedade. Tais resultados apontam para a relevância do desenvolvimento de estratégias e pesquisas com a finalidade de promover a reabilitação de trabalhadores com coronariopatia para que possam retornar mais rapidamente a suas atividades.

\section{Contribuições de autoria}

Uhlmann DB e Alexandre NMC participaram na concepção do projeto e, juntamente com Rodrigues RCM e Moreira São-João T, no delineamento do estudo, na coleta, análise e interpretação dos dados, na elaboração e revisão crítica do manuscrito e na aprovação da sua versão final publicada. Todas as autoras assumem integral responsabilidade pelo trabalho e o conteúdo publicado. 


\section{Referências}

1. World Health Organization. Cardiovascular diseases (CVDs) [Internet]. Geneva: WHO; 2016 [acesso em 14 ago 2019]. Disponível em: http:// www.who.int/mediacentre/factsheets/fs317/en/

2. Isaaz K, Coudrot M, Sabry MH, Cerisier A, Lamaud M, Robin C, et al. Return to work after acute ST-segment elevation myocardial infarction in the modern era of reperfusion by direct percutaneous coronary intervention. Arch Cardiovasc Dis. 2010;103(5):310-6.

3. Brink E, Brandstrom Y, Cliffordsson C, Herlitz J, Karlson BW. Illness consequences after myocardial infarction: problems with physical functioning and return to work. J Adv Nurs. 2008;64(6):587-94.

4. Slebus FG, Jorstad HT, Peters RJ, Kuijer PPFM, Willems JHHBM, Sluiter JK, et al. Return to work after an acute coronary syndrome: patients' perspective. Saf Health Work. 2012;3(2):117-22.

5. Waszkowska M, Szymczak W. Return to work after myocardial infarction: a retrospective study. Int J Occup Med Environ Health. 2009;22(4):373-81.

6. Anjos DBM, Rodrigues RCM, Padilha KM, Pedrosa RBS, Gallani MCBJ. Influence of sociodemographic and clinical characteristics at the impact of valvular heart disease. Rev Bras Enferm. 2016;69(1):40-6.

7. Organização Mundial da Saúde. Classificação internacional de funcionalidade, incapacidade e saúde - CIF. Lisboa: OMS; 2004 [acesso em 14 ago 2019]. Disponível em: https://catalogo.inr.pt/ documents/11257/0/CIF + 2004

8. Gallasch CH, Alexandre NMC, Esteves SCB. Propriedades psicométricas do Questionário de Avaliação de Desempenho no Trabalho em trabalhadores submetidos à radioterapia. Rev Enferm UERJ. 2015;23(6):817-24.

9. Gallasch CH, Alexandre NMC, Amick B. Crosscultural adaptation, reliability, and validity of the work role functioning questionnaire to Brazilian Portuguese. J Occup Rehabil. 2007;17(4):701-11.

10. Pfeiffer E. A short portable mental status questionnaire for the assessment of organic brain deficit in elderly patients. J Am Geriatr Soc. 1975;23(10):433-41.

11. Mendez RDR, Rodrigues RCM, Cornélio ME, Gallani MCBJ, Godin G. Desenvolvimento de instrumento para medida dos fatores psicossociais determinantes do comportamento de atividade física em coronariopatas. Rev Esc Enferm USP. 2010;44(3):584-96.

12. Silva SM, Baptista PCP. A incapacidade vivenciada por trabalhadores de enfermagem no retorno ao trabalho. Cienc Cuid Saude. 2013;12(3):522-8.

13. Hébert F. Les indicateurs de lésions en santé et sécurité du travail: analyse par secteur d'activité économique en 1991. Montréal: Institut de Recherche Robert-Sauvé en santé et sécurité au travail; 1996.

14. Domingues GBL, Gallani MCBJ, Gobatto CA, Miura CTP, Rodrigues RCM, Myers J. Cultural adaptation of an instrument to assess physical fitness in cardiac patients. Rev Saude Publica. 2011;45(2):276-85.

15. Oldridge N, Guyatt G, Jones N, Crowe J, Singer J, Feeny D, et al. Effects on quality of life with comprehensive rehabilitation after acute myocardial infarction. Am J Cardiol. 1991;67:1084-9.

16. Benetti M, Nahas MV, Barros MVG. Reproducibility and validity of a Brazilian version of the MacNew quality of life after myocardial infarction (MacNew QLMI) questionnaire. Med Sci Sports Exerc. 2001;33(5):S62.

17. Botega NJ, Pondé MP, Medeiros PL, Lima MG, Guerreiro CAM. Validação da escala hospitalar de ansiedade e depressão em pacientes epiléticos ambulatoriais. J Bras Psiquiatr. 1998;47(6):285-9.

18. Lisspers J, Nygren A, Söderman E. Hospital Anxiety and Depression Scale (HAD): some psychometric data for a Swedish sample. Acta Psychiatr Scand. 1997;96(3):281-6.

19. Zigmond AS, Snaith RP. The hospital anxiety and depression scale. Acta Psychiatr Scand. 1983;67(6):361-70.

20. Padilha KM, Gallani MCBJ, Colombo RCR. Development of an instrument to measure beliefs and attitudes from heart valve disease patients. Rev Latinoam Enferm. 2004;12(3):453-59.

21. Santos RAB, Rodrigues RCM, Padilha KM, Rodrigues SLL, Spana TM, Gallani MCBJ. Validation of an instrument to measure the impact of coronary disease on patient's daily life. J Clin Nurs. 2012;21(3-4):485-94.

22. Pagano M, Gauvreau K. Princípios de bioestatística. São Paulo: Cengage Learning; 2004.

23. Cohen J. Statistical power analysis for the behavioral sciences. 2nd ed. Mahwah: Lawrence Erlbaum Associates; 1988.

24. Lourenço LBA, Rodrigues RCM, Ciol MA, SãoJoão TM, Cornélio ME, Dantas RAS, et al. A randomized controlled trial of the effectiveness of planning strategies in the adherence to medication for coronary artery disease. J Adv Nurs. 2014;70(7):1616-28.

25. Spana TM, Rodrigues RCM, Gallani MCBJ, Mendez RDR. Comportamiento de la actividade física en la miocardipatía isquémica según el perfíl sociodemográfico y clínico. Rev Bras Enferm. 2010;63(5):741-8.

26. Saldanha JHS, Pereira APM, Neves RF, Lima MAG. Facilitadores e barreiras de retorno ao trabalho 
de trabalhadores acometidos por LER/DORT. Rev Bras Saude Ocup. 2013;38(127):122-38.

27. Maranhão Neto GA, Leon ACP, Farinatti PTV. Validade e equivalência da versão em português do Veterans Specific Activity Questionnaire. Arq Bras Cardiol. 2011;97(2):130-5.

28. Lourenço LBA, Rodrigues RCM, São-João TM, Gallani MC, Cornélio ME. Qualidade de vida de coronariopatas após implementação de estratégias de planejamento para adesão medicamentosa. Rev Latinoam Enferm. 2015;23(1):11-9.

29. Moryś JM, Bellwon J, Höfer S, Rynkiewicz A, Gruchała, M. Quality of life in patients with coronary heart disease after myocardial infarction and with ischemic heart failure. Arch Med Sci. 2016;12(2):326-33.
30. Nakajima KM, Rodrigues RCM, Gallani MCBJ, Alexandre NMC, Oldridge N. Psychometric properties of MacNew Heart Disease Healthrelated Quality of Life Questionnaire: Brazilian version. J Adv Nurs. 2009;65(5):1084-94.

31. Rodrigues SLL, Rodrigues RCM, São-João TM, Pavan RBB, Padilha KM, Gallani MC. Impact of the disease: acceptability, ceiling and floor effects and reliability of an instrument on heart failure. Rev Esc Enferm USP. 2013;47(5):1090-7.

32. O’Neil A, Sanderson K, Oldenburg B. Depression as a predictor of work resumption following myocardial infarction (MI): a review of recent research evidence. Health Qual Life Outcomes. 2010;8(1):95. 